\title{
The stability of the Kronecker product of Schur functions
}

\author{
Emmanuel Briand ${ }^{1}$, Rosa Orellana ${ }^{2}$ and Mercedes Rosas ${ }^{3}$ \\ ${ }^{1}$ Departamento de Matemática Aplicada I, Universidad de Sevilla, Escuela Técnica Superior de Ingeniería In- \\ formática, Avda. Reina Mercedes S/N, 41012 Sevilla, Spain. \\ ${ }^{2}$ Dartmouth College, Mathematics Department, 6188 Kemeny Hall, Hanover, NH, 03755, USA. \\ ${ }^{3}$ Departamento de Álgebra, Universidad de Sevilla, Aptdo. de Correos 1160, 41080 Sevilla, Spain.
}

\begin{abstract}
In the late 1930's Murnaghan discovered the existence of a stabilization phenomenon for the Kronecker product of Schur functions. For $n$ large enough, the values of the Kronecker coefficients appearing in the product of two Schur functions of degree $n$ do not depend on the first part of the indexing partitions, but only on the values of their remaining parts. We compute the exact value of $n$ when this stable expansion is reached. We also compute two new bounds for the stabilization of a particular coefficient of such a product. Given partitions $\alpha$ and $\beta$, we give bounds for all the parts of any partition $\gamma$ such that the corresponding Kronecker coefficient is nonzero. Finally, we also show that the reduced Kronecker coefficients are structure coefficients for the Heisenberg product introduced by Aguiar, Ferrer and Moreira.
\end{abstract}

Résumé. Dans les années 30 Murnaghan a découvert une propriété de stabilité pour le produit de Kronecker de fonctions de Schur. En degré assez grand, les valeurs des coefficients qui aparaissent dans le produit de Kronecker de deux fonctions de Schur ne dépendent pas de la première part des partitions en indice, mais seulement des parts suivantes. Dans ce travail nous calculons la valeur exacte du degré partir duquel ce développement stable est atteint. Nous calculons aussi deux nouvelles bornes supérieures pour la stabilisation d'un coefficient particulier d'un tel produit. Nous donnons en outre, pour $\alpha$ et $\beta$ fixés, des bornes supérieures pour toutes les parts des partition $\gamma$ rendant le coefficient de Kronecker d'indices $\alpha, \beta, \gamma$ non-nul. Finalement, nous identifions les coefficients de Kronecker réduits comme des constantes de structures pour le produit de Heisenberg de fonctions symétriques défini par Aguiar, Ferrer et Moreira.

Resumen. Hace poco más de 80 años Murnaghan descubrió un fenómeno de estabilidad para el producto de Kronecker de dos funciones de Schur. En grado suficientemente grande, los valores de los coeficientes de Kronecker que aparecen en el producto de Kronecker de dos funciones de Schur, no dependen de las primeras partes de las particiones que las indexan, sino solamente de sus demás partes. En este trabajo calculamos exactemente cuando este desarrollo estable esta alcanzado. También calculamos dos nuevas cotas para que cualquier familia dada de coeficientes de Kronecker se estabilice. Dadas dos particiones $\alpha$ y $\beta$, proporcionamos cotas superiores para todas las partes de cualquier partición $\gamma$ tal que el coeficiente de Kronecker correspondiente no sea nulo. Finalmente, identificamos los coeficientes de Kronecker reducidos como constantes de estructura del producto de Heisenberg de funciones simétricas, introducido por Aguiar, Ferrer y Moreira.

Keywords: Symmetric functions, Kronecker coefficients 


\section{Introduction}

The understanding of the Kronecker coefficients of the symmetric group $g_{\alpha, \beta}^{\gamma}$ (the multiplicities appearing when the tensor product of two irreducible representations of the symmetric group is decomposed into irreducibles; equivalently, the structural constants for the Kronecker product $*$ of symmetric functions in the basis of Schur functions, $s_{\lambda}$ ) is a longstanding open problem. Richard Stanley writes "One of the main problems in the combinatorial representation theory of the symmetric group is to obtain a combinatorial interpretation for the Kronecker coefficients" [30]. It is also a source of new challenges such as the problem of describing the set of non-zero Kronecker coefficients [28], a problem inherited from quantum information theory [18, 10]. Or proving that the positivity of a Kronecker coefficient can be decided in polynomial time, a problem posed by Mulmuley at the heart of his Geometric Complexity Theory [24] (see also the introductory paper by Bürgisser, Landsberg, Manivel and Weyman [2]).

In our work we study in more detail a remarkable stability property for the Kronecker products of Schur functions discovered by Murnaghan [26, 27]. This property is best shown on an example. Consider the Kronecker products $s_{(n-2,2)} * s_{(n-2,2)}$ :

$$
\begin{aligned}
& s_{2,2} * s_{2,2}=s_{4}+s_{1,1,1,1}+s_{2,2} \\
& s_{3,2} * s_{3,2}=s_{5}+s_{2,1,1,1}+s_{3,2}+s_{4,1}+s_{3,1,1}+s_{2,2,1} \\
& s_{4,2} * s_{4,2}=s_{6}+s_{3,1,1,1}+2 s_{4,2}+s_{5,1}+s_{4,1,1}+2 s_{3,2,1}+s_{2,2,2} \\
& s_{5,2} * s_{5,2}=s_{7}+s_{4,1,1,1}+2 s_{5,2}+s_{6,1}+s_{5,1,1}+2 s_{4,2,1}+s_{3,2,2}+s_{4,3}+s_{3,3,1} \\
& s_{6,2} * s_{6,2}=s_{8}+s_{5,1,1,1}+2 s_{6,2}+s_{7,1}+s_{6,1,1}+2 s_{5,2,1}+s_{4,2,2}+s_{5,3}+s_{4,3,1}+s_{4,4} \\
& s_{7,2} * s_{7,2}=s_{9}+s_{6,1,1,1}+2 s_{7,2}+s_{8,1}+s_{7,1,1}+2 s_{6,2,1}+s_{5,2,2}+s_{6,3}+s_{5,3,1}+s_{5,4}
\end{aligned}
$$

And, actually, in all degree $n \geq 8$ we have the expansion:

$$
s_{\bullet, 2} * s_{\bullet, 2}=s_{\bullet}+s_{\bullet, 1,1,1}+2 s_{\bullet, 2}+s_{\bullet, 1}+s_{\bullet, 1,1}+2 s_{\bullet, 2,1}+s_{\bullet, 2,2}+s_{\bullet, 3}+s_{\bullet, 3,1}+s_{\bullet, 4}
$$

For $\alpha$ partition and $n$ integer, set $\alpha[n]$ for $\left(n-|\alpha|, \alpha_{1}, \alpha_{2}, \ldots\right)$. Murnaghan's general result is that for any partitions $\alpha$ and $\beta$, the expansions of $s_{\alpha[n]} * s_{\beta[n]}$ in the Schur basis all coincide for $n$ big enough, except for the first part of the indexing partitions (which is determined by the degree $n$ ). This implies in particular that given any three partitions $\alpha, \beta$ and $\gamma$, the sequence of Kronecker coefficients $g_{\alpha[n] \beta[n]}^{\gamma[n]}$ is eventually constant. The reduced Kronecker coefficient $\bar{g}_{\alpha, \beta}^{\gamma}$ is defined as the stable value of this sequence. In our example, we see that $\bar{g}_{(2),(2)}^{(2)}=2$ and $\bar{g}_{(2),(2)}^{(4)}=1$.

When does a Kronecker product $s_{\alpha[n]} * s_{\beta[n]}$ stabilizes? When does a sequence of Kronecker coefficients $g_{\alpha[n] \beta[n]}^{\gamma[n]}$ becomes constant? Interestingly, these questions lead to look for linear inequalities fulfilled by the sets of triples of partitions $(\alpha, \beta, \gamma)$ whose corresponding reduced Kronecker coefficient $\bar{g}_{\alpha, \beta}^{\gamma}$ is non-zero. The analogous problem for Kronecker coefficients is of major importance, see [18, 28].

In view of the difficulty of studying the Kronecker coefficients, it is surprising to obtain theorems that hold in general. Regardless of this, we present new results of a general nature.

We find an elegant expression for the precise degree $n=\operatorname{stab}(\alpha, \beta)$ at which the expansion of the Kronecker product $s_{\alpha[n]} * s_{\beta[n]}$ stabilizes:

$$
\operatorname{stab}(\alpha, \beta)=|\alpha|+|\beta|+\alpha_{1}+\beta_{1}
$$


Using Weyl's inequalities [35] for eigenvalues of triples of hermitian matrices fulfilling $A+B=C$, we find the maximum of $\gamma_{1}$ and upper bounds for all parts $\gamma_{k}$, among all $\gamma$ in $\operatorname{Supp}(\alpha, \beta)=\left\{\gamma: \bar{g}_{\alpha, \beta}^{\gamma}>0\right\}$.

Finally, we find upper bounds for the index $n=\operatorname{stab}(\alpha, \beta, \gamma)$ at which the sequence $g_{\alpha[n] \beta[n]}^{\gamma[n]}$ becomes constant, improving previously known bounds due to Brion [9] and Vallejo [34].

Detailed proofs for the results presented in this extended abstract can be found in [7].

\section{Preliminaries}

We assume that the reader is familar with the basic definitions in the theory of symmetric funcion, see [21] or [30].

Let $\lambda$ be a partition of $n$. Let $V_{\lambda}$ the irreducible representation of the symmetric group $\mathfrak{S}_{n}$ indexed by $\lambda$. The Kronecker coefficient $g_{\mu, \nu}^{\lambda}$ is the multiplicity of $V_{\lambda}$ in the decomposition into irreducible representations of the tensor product $V_{\mu} \otimes V_{\nu}$. The Frobenius map identify $V_{\lambda}$ with the Schur function $s_{\lambda}$. In doing so, it allows us to lift the tensor product of representations of the symmetric group to the setting of symmetric functions. Accordingly, the Kronecker coefficients $g_{\mu, \nu}^{\lambda}$ define the Kronecker product on symmetric functions by setting

$$
s_{\mu} * s_{\nu}=\sum_{\lambda} g_{\mu, \nu}^{\lambda} s_{\lambda}
$$

We use the Jacobi-Trudi determinant to extend the definition of $s_{\mu}$ to the case where $\mu$ is any finite sequence of $n$ integers :

$$
s_{\mu}=\operatorname{det}\left(h_{\mu_{j}+i-j}\right)_{1 \leq i, j \leq n},
$$

where $h_{k}$ is the complete homogeneous symmetric function of degree $k$. In particular, $h_{k}=0$ if $k$ is negative, and $h_{0}=1$. It is not hard to see that such a Jacobi-Trudi determinant $s_{\mu}$ is either zero or \pm 1 times a Schur function.

The starting point of our investigations is a beautiful theorem of Murnaghan. Given a partition $\lambda=$ $\left(\lambda_{1}, \lambda_{2}, \ldots\right)$ and an integer $n$, we denote by $\lambda[n]$ the sequence $\left(n-|\lambda|, \lambda_{1}, \lambda_{2}, \ldots\right)$. Notice that $\lambda[n]$ is a partition only if $n-|\lambda| \geq \lambda_{1}$.

Murnaghan Theorem (Murnaghan, [26, 27]). There exists a family of non-negative integers $\left(\bar{g}_{\alpha \beta}^{\gamma}\right)$ indexed by triples of partitions $(\alpha, \beta, \gamma)$ such that, for $\alpha$ and $\beta$ fixed, only finitely many terms $\bar{g}_{\alpha \beta}^{\gamma}$ are nonzero, and for all $n \geq 0$,

$$
s_{\alpha[n]} * s_{\beta[n]}=\sum_{\gamma} \bar{g}_{\alpha \beta}^{\gamma} s_{\gamma[n]}
$$

Moreover, the coefficient $\bar{g}_{\alpha \beta}^{\gamma}$ vanishes unless the weights of the three partitions fulfill the inequalities:

$$
|\alpha| \leq|\beta|+|\gamma|, \quad|\beta| \leq|\alpha|+|\gamma|, \quad|\gamma| \leq|\alpha|+|\beta| .
$$

In what follows, we refer to these inequalities as Murnaghan's inequalities. We follow Klyachko [18] and call the coefficients $\bar{g}_{\alpha \beta}^{\gamma}$ the reduced Kronecker coefficients. An elegant proof of Murnaghan's Theorem, using vertex operators on symmetric functions, is given in [33].

Example 1. According to Murnaghan's theorem the reduced Kronecker coefficients determine the Kronecker product of two Schur functions, even for small values of $n$. For instance,

$$
s_{2,2} * s_{2,2}=s_{4}+s_{1,1,1,1}+2 s_{2,2}+s_{3,1}+s_{2,1,1}+2 s_{1,2,1}+s_{0,2,2}+s_{1,3}+s_{0,3,1}+s_{0,4}
$$


The Jacobi-Trudi determinants corresponding to $s_{1,2,1}$ and $s_{0,2,2}$ have a repeated column, hence they are zero. On the other hand, it is easy to see that $s_{1,3}=-s_{2,2}, s_{0,3,1}=-s_{2,1,1}$, and $s_{0,4}=-s_{3,1}$. After taking into account the resulting cancellations, we recover the expression of the Kronecker product $s_{2,2} * s_{2,2}$ in the Schur basis: $s_{4}+s_{1,1,1,1}+s_{2,2}$.

The reduced Kronecker coefficients contain the Littlewood-Richardson coefficients as special cases, as it was observed already by Murnaghan [27] and Littlewood [20]. Precisely, if $|\gamma|=|\alpha|+|\beta|$, then the reduced Kronecker coefficient $\bar{g}_{\alpha, \beta}^{\gamma}$ is equal to the Littlewood-Richardson coefficient $c_{\alpha, \beta}^{\gamma}$.

\section{Recovering the Kronecker coefficients from reduced Kronecker coefficients}

By definition, the reduced Kronecker coefficients are particular instances of Kronecker coefficients. We show that the reduced Kronecker coefficients contain enough information to recover exact value of the Kronecker coefficients. Let $u=\left(u_{1}, u_{2}, \ldots\right)$ be an infinite sequence and $i$ a positive integer. Define $u^{\dagger i}$ as the sequence obtained from $u$ by adding 1 to its $i-1$ first terms and erasing its $i$-th term:

$$
u^{\dagger i}=\left(1+u_{1}, 1+u_{2}, \ldots, 1+u_{i-1}+1, u_{i+1}, u_{i+2}, \ldots\right)
$$

Partitions are identified with infinite sequences by appending trailing zeros. Under this identification, when $\lambda$ is a partition then so is $\lambda^{\dagger i}$ for all positive $i$.

Theorem 2.1 (Computing the Kronecker coefficients from the reduced Kronecker coefficients). Let $n$ be a nonnegative integer and $\lambda, \mu$, and $\nu$ be partitions of $n$. Then

$$
g_{\mu \nu}^{\lambda}=\sum_{i=1}^{\ell(\mu) \ell(\nu)}(-1)^{i+1} \bar{g}_{\bar{\mu}}^{\lambda^{\dagger i}}
$$

\section{The stabilization of the Kronecker products}

Let us define here formally $\operatorname{stab}(\alpha, \beta)$. Let $V$ be the linear operator on symmetric functions defined on the Schur basis by $V\left(s_{\lambda}\right)=s_{\lambda+(1)}$ for all partitions $\lambda$.

Definition $(\operatorname{stab}(\alpha, \beta))$. Let $\alpha$ and $\beta$ be partitions. Then $\operatorname{stab}(\alpha, \beta)$ is defined as the smallest integer $n$ such that

$$
s_{\alpha[n+k]} * s_{\beta[n+k]}=V^{k}\left(s_{\alpha[n]} * s_{\beta[n]}\right)
$$

for all $k>0$.

As an illustration see the example in the introduction where $\alpha=\beta=(2)$ and the Kronecker product is stable starting at $s_{(6,2)} * s_{(6,2)}$. Since $(6,2)$ is a partition of 8 , we get that $\operatorname{stab}(\alpha, \beta)=8$.

Theorem 3.1. Let $\alpha$ and $\beta$ be two partitions. Then

$$
\operatorname{stab}(\alpha, \beta)=|\alpha|+|\beta|+\alpha_{1}+\beta_{1} .
$$

To show that this theorem holds, we first reduce the calculation of $\operatorname{stab}(\alpha, \beta)$ to maximizing the linear form $|\gamma|+\gamma_{1}$ on $\operatorname{Supp}(\alpha, \beta)$

$$
\operatorname{stab}(\alpha, \beta)=\max \left\{|\gamma|+\gamma_{1} \mid \gamma \text { partition, } \bar{g}_{\alpha, \beta}^{\gamma}>0\right\} .
$$


Then, we use the following formula that gives a decomposition of $\bar{g}_{\alpha \beta}^{\gamma}$ as a sum of nonnegative summands obtained from a formula due to Littlewood to show that $\max \left\{|\gamma|+\gamma_{1} \mid \gamma\right.$ partition, $\left.\bar{g}_{\alpha, \beta}^{\gamma}>0\right\}=|\alpha|+$ $|\beta|+\alpha_{1}+\beta_{1}$.

Let $c_{\alpha, \beta, \gamma}^{\delta}$ be the coefficient of $s_{\delta}$ in the product $s_{\alpha} s_{\beta} s_{\gamma}$.

Lemma 3.2. Let $\alpha, \beta$, $\gamma$ be partitions. Then,

$$
\bar{g}_{\alpha, \beta}^{\gamma}=\sum g_{\delta, \epsilon}^{\zeta} c_{\delta, \sigma, \tau}^{\alpha} c_{\epsilon, \rho, \tau}^{\beta} c_{\zeta, \rho, \sigma}^{\gamma}
$$

\section{Row lengths for partitions indexing nonzero Kronecker coeffi- cients.}

In this section we give bounds for row lengths of partitions indexing nonzero Kronecker coefficients. We begin by reminding the reader about the powerful result:

Proposition 4.1 ( Klemm [17], Dvir [13] Theorem 1.6, Clausen and Meier [11] Satz 1.1.). Let $\alpha$ and $\beta$ be partitions of the same weight. Then,

$$
\max \left\{\gamma_{1} \mid \gamma \text { partition s. t. } g_{\alpha, \beta}^{\gamma}>0\right\}=|\alpha \cap \beta|
$$

where $\alpha \cap \beta=\left(\min \left(\alpha_{1}, \beta_{1}\right), \min \left(\alpha_{2}, \beta_{2}\right), \ldots\right)$.

Proposition 4.1 is the inspiration for some of the results in this section. Two closely related questions come to mind: First, can we prove an analogous result for the reduced Kronecker coefficients? Second, what can be said about the remaining parts of a partition $\gamma$ such that $g_{\alpha, \beta}^{\gamma}>0$ (or similarly, such that $\left.\bar{g}_{\alpha, \beta}^{\gamma}>0\right)$ ?

We answer the first question in the affirmative by showing that

Theorem 4.2. Let $\alpha$ and $\beta$ be partitions. Then,

$$
\max \left\{\gamma_{1} \mid \gamma \text { partition, } \bar{g}_{\alpha, \beta}^{\gamma}>0\right\}=|\alpha \cap \beta|+\max \left(\alpha_{1}, \beta_{1}\right)
$$

We also obtained a set of bounds for the remaing parts of such a $\gamma$ using Weyl's inequalities triples of spectra of hermitian matrices fulfilling $A+B=C$ [35]. This bounds are known to hold as well for the indices of the non-zero Littlewood-Richardson coefficients (see for instance [14]).

Theorem 4.3. Let $\alpha$ and $\beta$ be partitions. If $\bar{g}_{\alpha, \beta}^{\gamma}>0$, then, for all positive integers $i, j$, we have that

$$
\gamma_{i+j-1} \leq\left|E_{i} \alpha \cap E_{j} \beta\right|+\alpha_{i}+\beta_{j}
$$

where $E_{k} \lambda$ stands for the partition obtained from $\lambda$ by erasing its $k$-th part.

Finally, combining Murnaghan's inequalities with Proposition 4.1 we obtain

$$
\begin{aligned}
& \max \left\{|\gamma| \mid \gamma \text { partition, } \bar{g}_{\alpha, \beta}^{\gamma}>0\right\}=|\alpha|+|\beta|, \\
& \min \left\{|\gamma| \mid \gamma \text { partition, } \bar{g}_{\alpha, \beta}^{\gamma}>0\right\}=\max (|\alpha|,|\beta|)-|\alpha \cap \beta| .
\end{aligned}
$$


The first equality readily implies that

$$
\gamma_{k} \leq \frac{|\alpha|+|\beta|}{k}
$$

Example 2. Let $\alpha=(2)$ and $\beta=(4,3,2)$, then the first row of the table are the nonzero values of $\gamma_{k}$ and the second row are the values predicted by equations $(6)$ and $(7)$ :

\begin{tabular}{l|l|l|l|l|l}
$k$ & 1 & 2 & 3 & 4 & 5 \\
\hline $\max$ values for $\gamma_{k}$ & 6 & 4 & 3 & 2 & 1 \\
\hline bound for $\gamma_{k}$ & 6 & 5 & 3 & 2 & 2
\end{tabular}

In the case that $\alpha=(3,1)$ and $\beta=(2,2)$ we get

\begin{tabular}{l|l|l|l|l|l|l}
$k$ & 1 & 2 & 3 & 4 & 5 & 6 \\
\hline $\max$ values for $\gamma_{k}$ & 6 & 3 & 2 & 1 & 1 & 1 \\
\hline bound for $\gamma_{k}$ & 6 & 4 & 2 & 2 & 1 & 1
\end{tabular}

These bounds also provide bounds for the non-zero Kronecker coefficients. Indeed, Michel Brion [9] showed that for any given $\alpha, \beta$ and $\gamma$, the sequence of the Kronecker coefficients $g_{\alpha[n], \beta[n]}^{\gamma[n]}$ is weakly increasing. As a consequence, $\bar{g}_{\alpha, \beta}^{\gamma}$ is non-zero whenever $g_{\alpha[n], \beta[n]}^{\gamma[n]}$ is non-zero for some $n$.

\section{The stabilization of the Kronecker coefficients}

In this section we study of a weaker version of the stabilization problem. One consequence of Murnaghan's Theorem is that each particular sequence of Kronecker coefficients $g_{\alpha[n], \beta[n]}^{\gamma[n]}$ stabilizes to $\bar{g}_{\alpha, \beta}^{\gamma}$, possibly before $n$ reaches $\operatorname{stab}(\alpha, \beta)$.

Definition $(\operatorname{stab}(\alpha, \beta, \gamma))$. Let $\alpha, \beta, \gamma$ be partitions. Then $\operatorname{stab}(\alpha, \beta, \gamma)$ is defined as the the smallest integer $N$ such that the sequences $\alpha[N], \beta[N]$ and $\gamma[N]$ are partitions and $g_{\alpha[n], \beta[n]}^{\gamma[n]}=\bar{g}_{\alpha, \beta}^{\gamma}$ for all $n \geq N$.

Two bounds have already been found for $\operatorname{stab}(\alpha, \beta, \gamma)$ by Brion [9] and Vallejo [34]. Brions' and Vallejo's bounds, respectively, are

$$
\begin{aligned}
& M_{B}(\alpha, \beta ; \gamma)=|\alpha|+|\beta|+\gamma_{1}, \\
& M_{V}(\alpha, \beta ; \gamma)=|\gamma|+ \begin{cases}\max \left\{|\alpha|+\alpha_{1}-1,|\beta|+\beta_{1}-1,|\gamma|\right\} & \text { if } \alpha \neq \beta \\
\max \left\{|\alpha|+\alpha_{1},|\gamma|\right\} & \text { if } \alpha=\beta\end{cases}
\end{aligned}
$$

Our first contribution is the following Lemma which describes a general technique for producing linear upper bounds for $\operatorname{stab}(\alpha, \beta, \gamma)$.

Lemma 5.1. Let $f$ be a function on triples of partitions such that for all $i$,

$$
f\left(\alpha, \beta, \gamma^{\dagger 1}\right) \geq f\left(\alpha, \beta, \gamma^{\dagger i}\right) .
$$

Set $\mathcal{M}_{f}(\alpha, \beta, \gamma)=|\gamma|+f(\alpha, \beta, \bar{\gamma})$ and assume also that whenever $\bar{g}_{\alpha, \beta}^{\gamma}>0$,

$$
\mathcal{M}_{f}(\alpha, \beta, \gamma) \geq \max \left(|\alpha|+\alpha_{1},|\beta|+\beta_{1},|\gamma|+\gamma_{1}\right) .
$$

Then whenever $\bar{g}_{\alpha, \beta}^{\gamma}>0$,

$$
\operatorname{stab}(\alpha, \beta, \gamma) \leq \mathcal{M}_{f}(\alpha, \beta, \gamma)
$$


Three functions $f$ such that $(8)$ holds have already appeared in this paper. Each one gives a bound for $\operatorname{stab}(\alpha, \beta, \gamma)$.

1. Murnaghan's triangle inequalities and Theorem 4.2 imply that (8) holds for $f(\alpha, \beta, \tau)=|\alpha|+|\beta|-$ $|\tau|$. Using our lemma, we recover Brion's bound.

2. From Theorem 4.2 we obtain that $(8)$ holds for $f(\alpha, \beta, \tau)=|\bar{\alpha} \cap \bar{\beta}|+\alpha_{1}+\beta_{1}$. In this situation, we obtain that $M_{1}(\alpha, \beta, \gamma)=|\gamma|+|\bar{\alpha} \cap \bar{\beta}|+\alpha_{1}+\beta_{1}$. From the symmetry of the Kronecker coefficients, we conclude that $\operatorname{stab}(\alpha, \beta, \gamma) \leq N_{1}(\alpha, \beta, \gamma)$ where

$$
N_{1}(\alpha, \beta, \gamma)=\min \left(M_{1}(\alpha, \beta, \gamma), M_{1}(\beta, \gamma, \alpha), M_{1}(\gamma, \alpha, \beta)\right)
$$

We have shown that $N_{1}$ improves both the bounds of Vallejo and of Brion.

3. Finally, Theorem 3.1 shows that $(8)$ holds for $f(\alpha, \beta, \tau)=1 / 2\left(|\alpha|+|\beta|+\alpha_{1}+\beta_{1}-|\tau|\right)$. Then $\operatorname{stab}(\alpha, \beta, \gamma) \leq N_{2}(\alpha, \beta, \gamma)$, where

$$
N_{2}(\alpha, \beta, \gamma)=\left[\frac{|\alpha|+|\beta|+|\gamma|+\alpha_{1}+\beta_{1}+\gamma_{1}}{2}\right]
$$

where $[x]$ denotes the integer part of $x$.

We conclude this section by applying our bounds to some interesting examples of Kronecker coefficients appearing in the literature.

Example 3 (The Kronecker coefficients indexed by three hooks). Our first example looks at the elegant situation where the three indexing partitions are hooks. Note that after deleting the first part of a hook we always obtain a one column shape. Let $\alpha=\left(1^{e}\right), \beta=\left(1^{f}\right)$ and $\gamma=\left(1^{d}\right)$ be the reduced partitions, with $d, e$ and $f$ positive. In Theorem 3 of [29], it was shown that Murnaghan's inequalities describe the stable value of the Kronecker coefficient $g_{\alpha[n], \beta[n]}^{\gamma[n]}$,

$$
\bar{g}_{\alpha, \beta}^{\gamma}=((e \leq d+f))((d \leq e+f))((f \leq e+d))
$$

where $((P))$ equals 1 if the proposition is true, and 0 if not.

Moreover, $\operatorname{stab}(\alpha, \beta, \gamma)$ was actually computed in the proof of Theorem 3 [29]. It was shown that the Kronecker coefficient equals 1 if and only if Murnaghan's inequalities hold, as well as the additional inequality $e+f \leq d+2(n-d)-2$. This last inequality says that:

$$
\operatorname{stab}(\alpha, \beta, \gamma)=\left[\frac{d+e+f+3}{2}\right]=N_{2}(\alpha, \beta, \gamma)
$$

To summarize, for triples of hooks, Murnaghan's inequalities govern the value of the reduced Kronecker coefficients, and $N_{2}$ is a sharp bound. On the other hand, the bounds provided by $N_{1}, N_{B}$, and $N_{V}$ are not in general sharp. 
Example 4 (The Kronecker coefficients indexed by two two-row shapes). After deleting the first part of a two-row partition we obtain a partition of length 1 . Let $\alpha$ and $\beta$ be one-row partitions. We have:

$$
\begin{aligned}
& N_{1}(\alpha, \beta, \gamma)=\alpha_{1}+\beta_{1}+\gamma_{1} \\
& N_{2}(\alpha, \beta, \gamma)=\alpha_{1}+\beta_{1}+\gamma_{1}+\left[\frac{\gamma_{2}+\gamma_{3}}{2}\right]
\end{aligned}
$$

It follows from [8] that when $\bar{g}_{\alpha, \beta}^{\gamma}>0$,

$$
\operatorname{stab}(\alpha, \beta, \gamma)=\gamma_{1}-\gamma_{3}+\alpha_{1}+\beta_{1}
$$

Neither $N_{1}$ nor $N_{2}$ are sharp bounds. Indeed, for $\bar{g}_{\alpha, \beta}^{\gamma}>0$ we have $\operatorname{stab}(\alpha, \beta, \gamma)<N_{1}$ if $\gamma_{3}>0$, and $\operatorname{stab}(\alpha, \beta, \gamma)<N_{2}$ if $\gamma_{2}>0$.

Moreover, $N_{1}<N_{2}$ when $\gamma_{2}+\gamma_{3}>1$.

Example 5 (The Kronecker coefficients: One of the partitions is a two-row shape). The case when $\gamma$ has only one row, $\gamma=(p)$, was studied in [4]. It was shown there (Theorem 5.1) that

$$
\operatorname{stab}(\alpha, \beta,(p)) \leq|\alpha|+\alpha_{1}+2 p
$$

\section{Further remarks on the reduced Kronecker coefficients}

There is strong evidence to believe that the reduced Kronecker coefficients are better behaved than the Kronecker coefficients and in some sense easier to study.

The saturation theorem of Terence Tao and Allen Knutson, imply that deciding whether a LittlewoodRichardson coefficient is positive can be done in polynomial time [23, 19, 12]. On the other hand, it is known that the Kronecker coefficients do not satisfy the saturation property. For example,

$$
g_{(n, n),(n, n)}^{(n, n)}=0 \text { if } n \text { is odd, but } g_{(n, n),(n, n)}^{(n, n)}=1 \text { if } n \text { is even. }
$$

This suggests that the Kronecker coefficients are harder to compute.

On the other hand, the reduced Kronecker coefficients are conjectured to satisfy the saturation property by Klyachko and Kirillov, [18, 16], and in a stronger form by King [15].

We believe that the study of the reduced Kronecker coefficients $\bar{g}_{\mu, \nu}^{\lambda}$ will lead to a better understanding of the Kronecker coefficients.

This paper is part of a series [6, 8] that studies the reduced Kronecker coefficients. Theorem 2.1 first appeared in [5], where it was used to compute the first explicit piecewise quasipolynomial description for the Kronecker coefficients indexed by two two-row shapes. That description was then used in [8] to test several conjectures of Mulmuley. As a result, we found counterexamples [6] for the strong version of his SH conjecture [24] on the behavior of the Kronecker coefficients under stretching of its indices. As pointed out by Ron King [15], our counterexample also implies that $Q_{\lambda, \mu}^{\nu}(t)=g_{t \lambda, t \mu}^{t \nu}$ is not an Ehrhart quasipolynomial. Therefore $Q_{\lambda, \mu}^{\nu}(t)$ can not count the number of integral points in any rational complex polytope. 
We have also found a very interesting connection to another product \# on symmetric functions, introduced by Aguiar, Ferrer and Moreira [1, 25] under the names smash or Heisenberg product, and independently, yet less explicitely, by Scharf, Thibon and Wybourne [32]. It fulfills

$$
f \# g=\sum f_{1} \cdot\left(f_{2} * g_{1}\right) \cdot g_{2}
$$

where $\Delta(f)=\sum f_{1} \otimes f_{2}$ and $\Delta(g)=\sum g_{1} \otimes g_{2}$ are in Sweedler's notation.

We have shown that the reduced Kronecker coefficients are the structure constants for this product in the basis $\left\{s_{\lambda}[\mathbb{X}-1]\right\}$ (the Schur functions at the alphabet $\mathbb{X}-1$, in the $\lambda$-ring notation). That is,

$$
s_{\alpha}[\mathbb{X}-1] \# s_{\beta}[\mathbb{X}-1]=\sum_{\gamma} \bar{g}_{\alpha, \beta}^{\gamma} s_{\gamma}[\mathbb{X}-1] .
$$

At this point, we hope that the reader is convinced that the reduced Kronecker coefficients are interesting objects on their own.

\section{Acknowledgments}

We thank Ernesto Vallejo for pointing to us the reference [9], Ron King for pointing to us Littlewood's formula, and Richard Stanley for suggesting to look at [16]. We also thank John Stembridge for making freely available his Maple package SF [31].

\section{References}

[1] Marcelo Aguiar, Walter Ferrer, and Walter Moreira. The smash product of symmetric functions. Extended abstract. ArXiv:math.CO/0412016, 2004.

[2] Peter Bürgisser, Joseph Landsberg, Laurent Manivel, and Jerzy Weyman. An overview of mathematical issues arising in the Geometric complexity theory approach to VP v.s. VNP.

ArXiv:0907.2850, 2009

[3] P. H. Butler and R. C. King. The symmetric group: characters, products and plethysms. J. Mathematical Phys., 14:1176-1183, 1973.

[4] Cristina M. Ballantine and Rosa C. Orellana. On the Kronecker product $s_{(n-p, p)} * s_{\lambda}$. Electronic Journal of Combinatorics, 12:\#R28, 26 pp. (electronic), 2005.

[5] Emmanuel Briand, Rosa Orellana, and Mercedes Rosas. Quasipolynomial formulas for the Kronecker coefficients indexed by two two-row shapes (extended abstract). ArXiv:0812.0861v1, 2008.

[6] Emmanuel Briand, Rosa Orellana, and Mercedes Rosas. Reduced Kronecker coefficients and counter-examples to Mulmuley's conjecture SH. ArXiv:0810.3163, 2008.

[7] Emmanuel Briand, Rosa Orellana, and Mercedes Rosas. On the stability of the Kronecker products of Schur functions. ArXiv:0907.4652, 2009.

[8] Emmanuel Briand, Rosa Orellana, and Mercedes Rosas. Quasipolynomial formulas for the Kronecker coefficients indexed by two two-row shapes. In preparation. 
[9] Michel Brion. Stable properties of plethysm: on two conjectures of Foulkes. Manuscripta Mathematica, 80:347-371, 1993.

[10] Matthias Christandl, Aram W. Harrow, and Graeme Mitchison. Nonzero Kronecker coefficients and what they tell us about spectra. Comm. Math. Phys., 270(3):575-585, 2007.

[11] Michael Clausen and Helga Meier. Extreme irreduzible Konstituenten in Tensordarstellungen symmetrischer Gruppen. Bayreuth. Math. Schr., 45:1-17, 1993.

[12] Jesús A. De Loera and Tyrrell B. McAllister. On the computation of Clebsch-Gordan coefficients and the dilation effect. Experiment. Math., 15(1):7-19, 2006.

[13] Yoav Dvir. On the Kronecker product of $S_{n}$ characters. J. Algebra, 154(1):125-140, 1993.

[14] William Fulton. Eigenvalues, invariant factors, highest weights, and Schubert calculus. Bull. Amer. Math. Soc. (N.S.), 37(3):209-249 (electronic), 2000.

[15] Ron King Some remarks on characters of symmetric groups, Schur functions, LittlewoodRichardson and Kronecker coefficients Workshop on the Mathematical Foundations of Quantum Information, Universidad de Sevilla, November 23-27 2009. http: // congreso.us .es/ enredo2009/Workshop.html

[16] Anatol N. Kirillov. An invitation to the generalized saturation conjecture. Publ. Res. Inst. Math. Sci., 40(4):1147-1239, 2004.

[17] Michael Klemm. Tensorprodukte von Charakteren der symmetrischen Gruppe. Arch. Math. (Basel), 28(5):455-459, 1977.

[18] Alexander Klyachko. Quantum marginal problem and representations of the symmetric group. ArXiv:quant-ph/0409113, september 2004.

[19] Allen Knutson and Terence Tao. Honeycombs and sums of Hermitian matrices. Notices Amer. Math. Soc., 48(2):175-186, 2001.

[20] D. E. Littlewood. Products and plethysms of characters with orthogonal, symplectic and symmetric groups. Canad. J. Math., 10:17-32, 1958.

[21] I. G. Macdonald. Symmetric functions and Hall polynomials. Oxford Mathematical Monographs. The Clarendon Press Oxford University Press, New York, second edition, 1995. With contributions by A. Zelevinsky, Oxford Science Publications.

[22] Laurent Manivel. On rectangular Kronecker coefficients. arXiv:0907.3351v1, July 2009.

[23] Ketan D. Mulmuley and Milind Sohoni. Geometric complexity theory III: on deciding positivity of Littlewood-Richardson coefficients. ArXiv:cs.CC/0501076, January 2005.

[24] Ketan D. Mulmuley. Geometric complexity theory VI: the flip via saturated and positive integer programming in representation theory and algebraic geometry. Technical Report TR-200704, Computer Science Department, The University of Chicago, may 2007. (GCT6). Available as ArXiv:cs/0704.0229 and at http://ramakrishnadas.cs.uchicago.edu. Revised version to be available here. 
[25] Walter Moreira Products of representations of the symmetric group and non-commutative versions. Ph.D. Thesis Texas A\&M University, 2008.

[26] Francis D. Murnaghan. The Analysis of the Kronecker Product of Irreducible Representations of the Symmetric Group. Amer. J. Math., 60(3):761-784, 1938.

[27] Francis D. Murnaghan. On the analysis of the Kronecker product of irreducible representations of $S_{n}$. Proc. Nat. Acad. Sci. U.S.A., 41:515-518, 1955.

[28] Luke Oeding. Report on "Geometry and representation theory of tensors for computer science, statistics and other areas.". ArXiv:0810.3940, 2008.

[29] Mercedes H. Rosas. The Kronecker product of Schur functions indexed by two-row shapes or hook shapes. Journal of algebraic combinatorics, 14(2):153-173, 2001. Previous version at ArXiv:math/0001084.

[30] Richard P. Stanley. Enumerative combinatorics. Vol. 2, volume 62 of Cambridge Studies in Advanced Mathematics. Cambridge University Press, Cambridge, 1999. With a foreword by GianCarlo Rota and appendix 1 by Sergey Fomin.

[31] John Stembridge. A Maple package for symmetric functions. Journal of Symbolic Computation, 20:755-768, 1995. The Maple package SF is available at http://www.math.lsa.umich. edu/ jrs/maple.html\#SF.

[32] Thomas Scharf, Jean-Yves Thibon, and Brian G. Wybourne. Reduced notation, inner plethysms and the symmetric group. J. Phys. A, 26(24):7461-7478, 1993.

[33] Jean-Yves Thibon. Hopf algebras of symmetric functions and tensor products of symmetric group representations. Internat. J. Algebra Comput., 1(2):207-221, 1991.

[34] Ernesto Vallejo. Stability of Kronecker products of irreducible characters of the symmetric group. Electronic journal of combinatorics, 6(1):1-7, 1999.

[35] Hermann Weyl. Das asymtotische Verteilungsgesetz der Eigenwerte lineare partialler Differentialgleichungen. Mathematische Annalen, 71:441-479, 1912. 
